\title{
Pseudotime analysis reveals novel regulatory factors for multigenic onset and monogenic transition of odorant receptor expression
}

Achim Tresch ( $\square$ achim.tresch@uni-koeln.de )

University of Cologne

Mohammad Hussainy

University of Cologne

Sigrun I. Korsching

University of Cologne

\section{Research Article}

Keywords:

Posted Date: February 23rd, 2022

DOI: https://doi.org/10.21203/rs.3.rs-1352877/v1

License: (c) (i) This work is licensed under a Creative Commons Attribution 4.0 International License.

Read Full License 


\section{Abstract}

During their maturation from horizontal basal stem cells, olfactory sensory neurons (OSNs) are known to select exactly one out of hundreds of olfactory receptors (ORs) and express it on their surface, a process called monogenic selection. Monogenic expression is preceded by a multigenic phase during which several OR genes are expressed in a single OSN. Here, we perform pseudotime analysis of a single cell RNA-Seq dataset of murine olfactory epithelium to precisely align the multigenic and monogenic expression phases with the cell types occurring during OSN differentiation. In combination with motif analysis of OR gene cluster-associated enhancer regions, we identify known and novel transcription (co-)factors (Ebf1, Lhx2, Ldb1, Fos and Sspp2) and chromatin remodelers (Kdm1a, Eed and Zmynd8) associated with $\mathrm{OR}$ expression. The inferred temporal order of their activity suggests novel mechanisms contributing to multigenic OR expression and monogenic selection.

\section{Introduction}

The sense of smell is tasked with the daunting challenge of making sense of potentially billions of different chemical stimuli to enable a multitude of different behaviors such as food search, prey hunting, predator evasion, mating and other social interactions ${ }^{1}$. This task is solved by several different receptor families, of which the best studied is both the evolutionary oldest and the largest (odorant receptor genes, OR genes) ${ }^{2}$. The analytical power of this system is maximal when information gathered from activation of individual receptors is kept separate at the peripheral level. Indeed for both vertebrates and insects it has been shown that individual olfactory sensory neurons (OSNs) express only a single OR gene out of the entire olfactory receptor repertoire, which has been christened as monogenic expression ${ }^{3,4}$. Sensory neurons expressing the same receptor are distributed across the olfactory sensory surface, but their axons converge into a single target region in the olfactory bulb, the first relay station of olfactory information processing ${ }^{5-8}$. These target regions (so-called glomeruli) show a stereotyped arrangement, resulting in a receptotopic map on the olfactory bulb (or antennal lobe in the case of insects). Thus monogenic expression has a central importance for the olfactory coding logic. In fact, expression of ORs is even monoallelic, i.e. restricted to one allele of the OR selected in monogenic expression $3,4,9,10$. The molecular path towards monogenic and monoallelic expression is still not well understood, and the relative timing of these processes is not clear.

To reach monogenic and monoallelic expression presents a massive challenge in the case of very large gene families such as those of mouse and rat ORs, which both number well over one thousand intact genes $^{2}$. A striking feature of genomic arrangement of OR genes is the occurrence in several clusters, which contain from a single to over one hundred different OR genes ${ }^{11}$. For mouse 68 such clusters have been identified, with the largest cluster containing 269 OR genes $^{11}$. Another large cluster contains all 145 class I OR genes, which show a spatially restricted expression pattern in the olfactory epithelium ${ }^{12-14}$. These observations have prompted the search for cluster-specific regulatory elements. In a seminal publication, 63 genomic regions containing such elements were identified and named after Greek 
islands ${ }^{11,15}$. Fourty-two class II OR clusters and the single class I OR cluster are associated with these Greek islands, which lie proximal to and sometimes even inside the clusters ${ }^{11}$. A common feature of Greek islands is the presence of closely adjacent Lhx2 and Ebf1-binding motifs, which are also found individually in promoter regions of individual OR genes ${ }^{16-19}$.

Beyond individual cluster-specific regulatory elements the chromatin structure itself appears to play an essential role in regulating OR expression. OSNs possess a unique nuclear architecture compared to other cell types including the basal cells giving rise to the OSN lineage. In the silent phase before onset of expression OR genes are aggregated in constitutive heterochromatin and are associated with its molecular hallmarks, $\mathrm{H} 3 \mathrm{~K} 9 \mathrm{me} 3$ and $\mathrm{H} 4 \mathrm{~K} 20 \mathrm{me} 3^{20,21}$. Onset of expression is concomitant with selective de-methylation (H3K9me3), methylation of H3K27me3 and re-location into expression-competent territory ${ }^{21-24}$. Moreover, of the two alleles of an active OR only one is found in the more plastic facultative heterochromatin ${ }^{22-24}$, i.e. amenable to expression, whereas the other remains blocked inside the constitutive heterochromatin, resulting in monoallelic expression. This suggests an involvement of chromatin remodelers in regulation of expression of OR genes. Furthermore, the stabilization of monogenic expression appears to require negative feedback from an active OR gene ${ }^{25,26}$, which may be mediated by silencing of the activating demethylase LSD1, synonym Kdm1 $\mathrm{a}^{27,28}$. Recent progress in deep sequencing techniques has allowed to obtain high quality single cell transcriptomes (scRNA-Seq), resulting in the surprising observation that monogenic expression of ORs found in mature OSNs is preceded by a multigenic phase in immature $\mathrm{OSN}^{29,30}$.

It is so far mostly unclear how these OR expression phases align to the developmental stages of OSN differentiation. Furthermore, although the basic stages (stem cell, dividing precursor cell, immature neuron, mature neuron) were known previously ${ }^{29,30}$, deep sequencing techniques allow an unbiased ordering of individual cells along pseudotime trajectories according to their entire transcriptome. This enables a more precise and more stringent categorization of developmental stages compared to previous attempts.

Here, we re-analyzed a scRNA-Seq dataset obtained by Fletcher et al ${ }^{31}$ with the goal to precisely determine the timing of multigenic and monogenic expression during OSN differentiation. A combination of sequence binding motif and time series analysis then identifies novel regulatory components involved in establishing OR gene expression patterns. We ascertain the transcription (co)factors and chromatin remodelers that are specifically correlated with the onset of multigenic and of monogenic expression (e.g., Fos, Ssbp2, Eed and Zmynd8). Finally, we suggest potential mechanisms for multigenic and monogenic selection.

\section{Results}

\section{Re-analysis of a single cell RNA-Seq data set reveals four lineages originating from globose basal cells.}


We re-analyzed a scRNA-Seq dataset obtained from Fletcher et $\mathrm{al}^{31}$. After quality filtering and preprocessing (Methods, SFigure 2,3), 687 cells were included into further analysis and grouped into 13 clusters using Seurat KNN clustering on the top 15 principal components (Methods). Dimension reduction and visualization was performed using principal components analysis (Figure 1A) and tSNE / UMAP. Using an extensive set of known marker genes, we assigned clusters to cell types of the main olfactory epithelium (MOE) (Figure 1B, Methods SFigure 4A). We detected all cell types described by Fletcher et $\mathrm{al}^{31}$, and additionally we could subdivide the globose basal stem cell cluster into quiescent cells (qGBC) and active cells (GBC). Active GBC were identified by their expression of Ascl1/Mash ${ }^{32-35}$ and by the presence of cell cycle genes such as Mki67 and Top2a. GBC are known as the adult OSN stem cells responsible for a sustained self-renewal of the OSNs throughout life ${ }^{35}$.

Further, trajectory inference by Slingshot ${ }^{36}$ (Methods) revealed a tree with four leaves (Figure 1A). Be aware that Slingshot does not provide information on the direction of development, but merely a tree topology. In the following, we restrict our analysis to the neuronal lineage and therefore choose qGBC as a root node for pseudotime analysis. qGBC has been reported as a general stem cell population in the MOE following injury ${ }^{35}$. A previous analysis by Fletcher et $\mathrm{al}^{31}$ focused on differentiation processes starting from $\mathrm{HBCO}$. They placed their root node in $\mathrm{HBCO}$, which is a leaf node of our tree, and therefore merely report three lineages. Apart from this, their reconstruction is essentially identical to ours. The four branches of our tree are (Figure 1A):

1) The basal stem cells lineage, which connects the quiescent horizontal basal stem cells (HBCO, represented by 91 cells in the data) via two transient populations of horizontal basal stem cells (HBC1 (29 cells) and HBC2 (118 cells)) with the qGBC (53 cells).

2) The supporting cells lineage ranges from mature sustentacular cells (mSus (70 cells)) to qGBCs, and includes immature sustentacular cells (iSus (49 cells)) and HBC2.

3) The microvillous cells lineage contains merely microvillous cells (MV (36 cells)). No transient cell types have been detected in this trajectory.

4) The neuronal lineage ends with mature olfactory sensory neurons (mOSN (32 cells)). It spans a range of several stages, namely GBC (34 cells), three intermediate neuronal precursors Early.INP (26 cells), Mid.INP (20 cells), Late.INP (34 cells) and immature olfactory sensory neurons (iOSN (95 cells)).

\section{OR gene expression is limited to the last three stages of OSN differentiation: Sudden onset of multigenic OR expression in Late.INP is followed by transition to monogenic expression in immature OSN stage.}


Expectedly, OR expression is essentially unique to the neuronal lineage (Figure 2). Next we used Slingshot to assign a pseudotime to each cell, thereby providing a linear order of all 294 cells in the neuronal lineage from qGBC to the terminal cell cluster (Methods). Our analysis could detect 157 cells of neuronal lineage that express at least one OR gene at relevant levels, i.e. $\geq 50$ normalized counts. 132 of them belonged to the last three stages of OSN differentiation. Most of Late.INP cells (28 of 34 ), iOSN (76 of 95 ) and mOSN (28 of 32) express at least one OR. Many cells express more than one OR (multigenic expression), in particular in the Late.INP stage (26 of 28 cells expressing OR genes, $92.8 \%$ ). The frequency of multigenic expression drops sharply in later stages, $42 \%$ and $32 \%$ for iOSN and mOSN, respectively. We found 212 different $O R$ genes that were expressed at least once in a single cell of the neuronal lineage (Excel file 1). Figure 2A shows the total number of reads that were assigned to $O R$ genes, separately for each cell. While aggregate OR expression levels are almost zero for qGBC/GBC, early and mid INPs, there is a steep onset of OR expression in Late.INP. Then, overall OR expression stays at similarly high levels in iOSN and mOSN (Figure 2A). A few OSN do not appear to express any OR at a relevant level. More precisely, 19 out 95 iOSN cells (20\%) and 4 out 32 mOSN $(12.5 \%)$ have less than 50 normalized OR counts. While it cannot be excluded that this is caused by incomplete annotation of the OR repertoire, it is also possible that reads were excluded due to multiple mapping to closely similar OR genes.

It is known that mature OSNs express only a single OR gene $3,4,9,37$, after a transient period of multigene expression $^{29,30}$. We therefore decided to rank OR genes by expression level, separately in each cell. We then investigated the temporal behavior of the top four ranked OR genes in each cell. These genes account for $99 \%$ of all reads $(413,388$ out of 416,033$)$ mapped to OR genes in cells from the neuronal lineage. The top-ranked gene of each stage will be referred to as 'winner' and the others as the 'runnersup'. While the abundance of all runners-up drops sharply after Late.INP, the winner does not drop and in fact absolute levels keep increasing several fold until the mOSN stage (Figure $2 \mathrm{C}, \mathrm{D}$ ). As a consequence the distance between winner and runners-up increases considerably in the iOSN stage and even more so in the mature neurons ( $\mathrm{mOSN}$ ). Since we observe each cell only once, we cannot be sure that the winner gene observed in one cell at a certain stage will be the highest expressed OR gene when that cell matures. However, this is by far the most plausible explanation, since a rank switch between the winner and a runner-up before / during iOSN stage would require a coordinated switch of expression between these two specific OR genes, from high to almost zero and vice versa, an unlikely scenario.

Taken together, the pseudotime analysis of neuronal lineage cells suggests three main phases for OR expression (Figure 2C,D):

1) The silent phase exhibits virtually no OR expression, which is the case for the four early stages of neuronal lineage (qGBC, GBC, Early.INP and Mid.INP). In the present data, the silent phase is represented by 133 cells.

2) The onset of OR expression (multigenic phase) is characterized by the simultaneous expression of several OR genes per cell at relatively similar levels; this phase is represented by 34 cells and contains 
specifically the Late.INP stage and the beginning of the iOSN stage.

3) Finally, the monogenic phase includes the end of the iOSN stage and the mOSN stage, where each cell expresses essentially one functional OR allele (henceforth called the "winner", Figure 2C) at a very high level while the remaining ORs (the "runners-up", Figure 2D) show no or very low expression. Our data contains 127 cells in this phase.

Our analysis reveals that Late.INP is a crucial stage in stochastic selection.

\section{OR gene expression during multigenic phase shows no sign of OR cluster-specific activation}

Next, in an attempt to infer the mechanism of activation in the multigenic stage and transition to monogenic stage, we analyzed the joint OR gene expression per cell (Figure 2B, Excel file 2). We found that each of the cells expressing more than one OR gene in the Late.INP stage had at least two active OR clusters (i.e., clusters with an OR gene expressed at a level of at least 50 counts). The number of active clusters reached up to 7 for some cells. We performed a permutation test to assess whether OR genes that are jointly active in one cell have the tendency to be located in the same cluster (see Scode 1 and the description therein). The results however show that the average number of clusters with more than 1 active OR gene is consistent with the null model of random cluster allocation of OR genes (SFigure 10, $p=0.112$ ). Moreover, we found that the top two active OR clusters (ranked by expression level) always belonged to different chromosomes in each cell of the Late.INP stage. Thus the onset of OR gene expression in the multigenic stage cannot be caused by activation of an individual chromosome or a particular OR cluster. Conversely the transition to monogenic stage could be partially caused by restriction of expression to a single chromosome and cluster. However, this may not be the only selection mechanism involved, as some cells express up to 3-4 different OR genes simultaneously within a single cluster. Specifically, for the 34 Late.INP cells we found 20 cells, which expressed at least 2 OR genes from the same cluster (expression defined as $\geq 50$ normalized counts). Thus, additional steps are required to restrict expression to a single gene within a cluster.

\section{Motif search in Greek island enhancers identifies novel transcription factors}

Next we proceeded to identify potential factors involved in both onset of multiple OR gene expression and the transition to monogenic expression. We focused on transcription factors that had a detectable expression in our scRNA-seq dataset. We consider a factor detectable if it has at least 35 counts in at least 15 out of 294 cells in the neuronal lineage, leaving us with 1358 (co)TFs.

Previously, 63 intergenic enhancer regions, termed Greek islands, have been identified inside or near OR clusters using DNase I hypersensitivity-sequencing and chromatin immunoprecipitation 
sequencing ${ }^{15}$ and ATAC-seq ${ }^{11}$. SFigure 1 shows the co-localization of the Greek islands and the OR clusters on a map of the murine genome. Chromatin conformation capture experiments have revealed that Greek islands extensively contact OR clusters, remarkably both in cis and trans ${ }^{38,39}$.

We performed a de novo motif search on all Greek island enhancer regions as annotated by ${ }^{11}$ using MEME $^{40,41}$ (Methods). Ungapped motif analysis of Greek islands identified one known motif, TYCCYWKGGGVCTHATTARM (reported in Monahan et $\mathrm{a}^{11}{ }^{11}$ ), and two novel motifs GVDHCYYCAGRGAV and TBYTCHTCTCYMCAGD GWBNY, with E-value $1.7 \mathrm{e}^{-057}, 3.7 \mathrm{e}^{-027}$ and $4.1 \mathrm{e}^{-008}$, respectively. Almost all Greek islands contain each of these motifs exactly once, except 8 Greek islands which are missing the third motif. TOMTOM was employed to align these motifs with known transcription factor motifs from the JASPAR database ${ }^{42}$ (Methods). TOMTOM did not predict any significant TF binding for the two novel motifs, therefore we do not discuss them further. We found 65 significant target binding sites for transcription factors inside the first motif (see Excel file 3). From those, 9 TFs were expressed at a detectable level.

The most significant motif, TYCCYWKGGGVCTHATTARM is composed of two adjacent submotifs, which are overlapped by a third submotif (Figure $3 \mathrm{~A}$ ). The first submotif is targeted by the COE1 DNA-binding domain which is found exclusively in the Ebf transcription factor family (Ebf1-4). The second submotif is bound by homeodomain TFs such as Lhx2, Emx2 and Uncx (Figure 3A). These results are consistent with previously reported Ebf1 and Lhx2 motifs to be positioned next to each other in most Greek islands ${ }^{11}$. Furthermore, the second submotif is expected to interact with transcription factors from three other families, the homeobox domain TF family, the Pou TF family (Pou6f1, this family has a strong enrichment in OR genes) and the ARID (AT-Rich Interaction Domain) domain TF family (Arid3a).

Noteworthy, our analysis predicted a third submotif, which is a possible binding site for Fos, Fosb and Fosl2. Fos and Fosb are well-known early response transcription factors, which in turn regulate a broad variety of other transcription factors thus regulating many physiological processes ${ }^{43-45}$. This Fos-binding motif overlaps with the end of the first and the beginning of the second submotif, suggesting a cooperative/inhibitory interaction of the respective binding factors. This possibility will be investigated further in the context of the pseudotime analysis.

Complementary to the de novo motif analysis, we did a forward motif search with $\mathrm{AME}^{46}$ looking for known TF binding sites enriched in the 63 Greek Islands (Methods). This returned a total of $120 \mathrm{TFs}$ (Excel file 4), of which 10 had a detectable expression (at least 35 count per cell in 15/294 cells of neuronal lineage) in our scRNA-seq dataset (SFigure 5). Six of those are also detected in the de novo motif search (see above), the additional TFs are Pax6, Dlx5, Pou2f1 and Arid3b (SFigure 3A). DIx5 is part of the same TF family as Lhx2, which was found in the de novo motif search. Pax6 and Pou2f1 have a homeobox domain, whereas Arid3b is part of the same TF family as Arid3a.

Taken together we describe 13 TFs that are found at detectable levels and predicted to bind to Greek islands by de novo and/or forward motif search. Next we determined the pseudotime profiles of these 
TFs and found clear and distinct temporal expression patterns, providing additional evidence for their active involvement in the regulation of OR expression.

\section{Pseudotime analysis suggests transcription factors involved in OR expression}

The sorting of cells according to pseudotime (Methods) generates, for each gene, a time course of its expression (see above). Notably, all TFs found by motif search in the previous paragraph show a pronounced temporal expression pattern, which belongs to one of three groups (Figure 3B and SFigure 5): The first group is active early in the silent phase, but strongly downregulated in late silent phase to reach a minimum in the multigenic phase (Fos, Fosb, Fosl2, Pax6 and Pou2f1). Some, but not all, are upregulated again in the monogenic phase (Fos, Fosb).The second group peaks within the multigenic phase (Ebf1, Lhx2, Emx2, Uncx and DIx5). The third group is specifically upregulated during the monogenic phase (Arid3a and Pou6f1). Hereafter we will refer to these group definitions.

The fact that all TFs with a known Greek island binding site show a clear temporal pattern prompted us to perform a systematic search for TFs that change their expression upon transition between the three phases of OR expression. We also include co-factors in this analysis, because co-factors such as LDB1 have been found to be selectively associated with Greek islands and were suggested to initiate OR expression ${ }^{38}$. We searched for (co-)TFs with a significant differential gene expression of at least 2-fold between silent vs. multigenic phase or between multigenic vs. monogenic phase (Methods), resulting in 83 (34 going up, 49 down) respectively 39 (8 up, 31 down) relevant hits (Figure 4A, see Excel files 5,6).

Several of these differentially expressed factors were also identified by motif analysis (e.g., Ebf1, Lhx2, DIx5, Fosb and Fos), but many are not. We manually inspected the pseudotime patterns of all differentially expressed (co-)TFs, and for detailed discussion, we selected those factors whose pseudotime expression pattern falls clearly into one of the three groups described above. We limit ourselves in the following to discussion of novel (co-)factors with additional evidence from motif analysis or a previous link to OR expression ${ }^{11,18,19,38}$. All other factors with a characteristic expression time course are shown in SFigure 9A.

For group 1 (active early in silent phase, downregulated in late silent phase and minimum in the multigenic phase) the differential expression search newly identified Egr1, whose expression resembles that of Fos and Fosb, which were already identified by motif analysis (see above). Therefore we searched explicitly for the known Egr1 binding motif (Methods) in Greek islands, which could be identified in 16 of 63 Greek islands. Fos, Fosb and Egr1 are immediate early genes, which are rapidly upregulated in response to external stimuli, immune response, and cellular stress ${ }^{45}$. Egr1, Fos and Fosb are specifically downregulated during the multigenic phase (Figure 4B and SFigure 9A). This suggests combinatorial interactions with the other components that regulate OR expression and will be discussed later. 
The second group peaks specifically within the multigenic phase and 6 factors have been identified by motif analysis (see above). Differential expression analysis further obtains the cofactor Ssbp2 (Figure 4B) and three factors, Cebpg, Rcor1 and Ldb1, which have been reported previously to be involved in OR expression ${ }^{11,38,47,48}$. Ssbp2 binds to Ldb1 and thereby prevents Ldb1 from degradation ${ }^{49,50}$. While Ldb1 and Lhx2 were shown to bind to Greek island enhancers to regulate OR expression in trans ${ }^{38}, \mathrm{Ssbp} 2$ is a novel candidate with such a function.

The third group is specifically upregulated during the monogenic phase and two factors from this group have been identified by motif analysis. Differential analysis identifies additionally the TFs Mef2b, Rfx3 and Sub1, also known as PC4 (Figure 4B and SFigure 9A). Among these factors, only Rfx3 has a known motif, for which we performed a strict motif search in Greek islands (Methods). We report that 56 out of 63 Greek islands contain the binding motif for Rfx3. Moreover, note that Mef2a, which shares a similar SRF binding domain with Mef $2 b^{51}$, is found to be strongly bound to OR promoters ${ }^{19}$.

Taken together, our pseudotime analysis recovers a large proportion of candidate (co-)TFs identified by motif analysis - both for initiating onset of OR expression, and for the transition to monogenic stage. Moreover it extends the range of candidates whose time course correlates with these two transitions, and consequently the regulatory repertoire for these transitions.

\section{Changes in chromatin remodeler expression accompany both transitions in the OR selection process}

It is known that chromatin changes accompany the selection of OR genes $20,21,23$. We therefore searched our data for chromatin remodelers that show expression changes during OR selection (Methods, Excel files 5,6). We confirmed previous observations that the chromatin remodelers Lbr and Cbx5 (SFigure 9B) are expressed at earlier stages and are downregulated in the course of OSN differentiation ${ }^{15,21}$. Furthermore, we discovered novel candidates for silencing OR genes, for onset of (multigenic) expression, and for transition to monogenic expression (Figure 4C):

Among the genes whose expression profiles fall into group 1 (minimum in multigenic phase), we found Eed, one of the constitutive subunits of the polycomb repressive complex 2, PRC2 (Figure 4C). Eed is required to maintain repressive $\mathrm{H} 3 \mathrm{~K} 27 \mathrm{me} 3$ marks $^{52,53}$ and its downregulation may lead to de-repression of OR expression in Late.INP stage. Note that another PRC2 subunit, Ezh2 is expressed during the silent phase as well, but decreases later, at the transition to monogenic phase (SFigure 6). Nsd1 is a histone methyltransferase that demethylates $\mathrm{H} 3 \mathrm{~K} 36 \mathrm{me} 2^{54}$ (Figure 4C). All remodelers found with a group 1 pseudotime profile (Hells, $\mathrm{H} 2 \mathrm{afz}$ and Set) are predicted to play a repressive role in the silent phase of OR selection (we only show H2afz as example SFigure 9B).

For group 2 (peak in multigenic phase), we found prominent chromatin remodelers such as Zmynd8, Ell3, Sertad2, Med12I and Scai (Figure 4C, SFigure 9B). We also investigated the expression profile of Kdm1a 
which was known before as a regulator of OR expression. Kdm1a alias LSD1 is a Lysine demethylase and functions both as a coactivator by demethylation of mono- or di-methylated $\mathrm{H} 3 \mathrm{~K} 9$ and as a corepressor through demethylation of mono- or di-methylated $\mathrm{H} 3 \mathrm{~K} 4^{55-58}$. There have been contradictory reports on the function of Kdm1a in OR expression as an activator ${ }^{27}$ or repressor of transcription ${ }^{48}$. The present data sheds light on this debate: While Kdm1 a expression sharply peaks directly before the multigenic phase (arguing for its role as activator), it can be part of the Co-REST repressor complex ${ }^{59}$. Two components of the Co-REST repressor complex, Rcor1 and Hdac2, sharply peak during multigenic phase (Figure 5C, SFigure 8A), arguing for a change of function of Kdm1a by recruitment to the Co-REST complex at the transition to monogenic phase $\mathrm{5}^{55,60,61}$.

Of the four novel remodelers with a group 2 pseudotime profile, Zmynd8 and Ell3 are highly differentially expressed (Figure 4C). Zmynd8, a chromatin reader, is a particularly appealing candidate, since it is also known to play a role in the selective expression of the immunoglobulin heavy chain (Igh) regions in immune cells (B cells). Its product ZMYND8 binds Igh super-enhancers known as 3' regulatory region (3'RR). ZMYND8 thereby controls the 3'RR activity by modulating the enhancer transcriptional status ${ }^{62}$. Consistent with an activating role during the multigenic phase, Ell3 does not only bind to active enhancers, but also marks the enhancers that are in a poised or inactive state in ES cells ${ }^{63}$.

Remodelers whose pseudotime profiles fall into group 3 (specifically upregulated during the monogenic phase) are Cbx4 (Chromobox 4) and Jarid2 (Figure 4C). Cbx4 is a component of a Polycomb group (PcG) multiprotein PRC1-like complex, which is required to maintain the transcriptionally repressive state of many genes ${ }^{64,65}$. Jarid2 (Jumonji and AT-Rich Interaction Domain Containing 2) is required to repress expression of cyclin-D1 (CCND1) in cardiac cells by setting H3K9 methylation marks ${ }^{66}$, and it is upregulated upon transition from Late.INP to iOSN (SFigure 4B), i.e. upon exit from the cell cycle.

So far, we identified several chromatin remodelers that add to the regulatory repertoire for the onset of OR expression, and for the transition to monogenic stage. Another important feature which requires chromatin remodeling is the monoallelic expression of ORs in mature OSNs. This feature appears to be established from the very beginning of OR expression ${ }^{4,23}$. Factors involved in generating allelic exclusion therefore would be expected to peak at least as early as factors regulating the onset of (multigenic) expression (group 2 factors). We found two remodeling factors with a very early onset within group 2 which could potentially play such a role: Smchd1 (structural maintenance of chromosomes flexible hinge domain-containing protein 1) and Cdy/2 (chromodomain Y-like protein 2) (see SFigure 7).

Based on the interactions and temporal coordination of remodelers and (co-)TFs, we generate and discuss some hypotheses about OR selection below.

\section{Discussion}

The monogenic expression of ORs presents a big challenge for the olfactory system, since it requires a random selection of exactly one OR per cell from the large family of OR genes. OR genes are known to be 
aggregated in silenced chromatin clusters during the silent phase, before the selection is made ${ }^{21}$. The identification of a multigenic state in early immature $O S N^{29,30}$ by scRNA-seq made clear that the escape from silencing is not limited to a single OR gene in a single cell. In a single cell, we observed up to seven different $\mathrm{OR}$ clusters and more than a dozen different OR genes concomitantly active. Both transcription factors and chromatin remodelers have been identified as regulators of OR expression.

Here we have employed pseudotime analysis of a single cell transcriptome data set ${ }^{31}$ focusing on OR expression. We aligned the three main phases of OR expression, silent, multigenic and monogenic ${ }^{29,30}$, with the stages of OSN differentiation as defined $i^{31}$. By analysis of winner vs. runners-up OR expression, we could precisely assign the onset of multigenic phase to Late.INP, and the onset of monogenic selection to iOSN. Most cells in the multigenic phase express ORs from more than one cluster and more than one chromosome (Figs. 2B, 5A). We tested whether ORs co-expressed in one cell tend to lie in identical OR clusters and did not find evidence for that (SCode 1, SFigure 10). We conclude that the selective expression of OR genes during multigenic phase is not caused by escape from compaction of merely one OR cluster. In the monogenic phase, the vast majority of cells express only a single OR gene (Fig. 5A).

We then identified candidate factors (TFs and cofactors including chromatin remodelers) differentially expressed between these stages and thus potentially involved in the transitions silent-to-multigenic (40 TFs, 43 cofactors) and multigenic-to-monogenic (19 TFs, 20 cofactors) (Methods, Excel files 5,6). Many of these differentially expressed factors are likely not directly involved in OR selection, since cells undergo many substantial changes along OSN differentiation. Thus, we performed an independent de novo motif analysis of Greek islands, which should enrich TFs involved in OR selection. This search revealed one motif that could be decomposed into three consecutive submotifs, only two of which were described previously ${ }^{11}$. We additionally recognized a central Fos binding motif overlapping the previously described two elements (Fig. 3A). All but one of the factors that bind to these motifs show characteristic pseudotime expression time courses, which could be classified in three groups (Fig. 5B). The temporal coordination of these factors together with the precise location of their respective binding sites enables us to generate hypotheses about their molecular interactions and possible functional consequences.

In the silent phase the transcriptional regulator Fos is binding to the central motif and may competitively prevent binding of the known activators of OR expression, Lhx2 and Ebf $1^{11}$, which bind to the left and right submotifs (Fig. 3A, top row of Fig. 5C). The strong downregulation of Fos expression in the multigenic phase then would allow Lhx2 and Ebf1 to access their binding motifs, and recruit their known binding partner $L d b 1$ to Greek islands ${ }^{38}$. Ldb1 mediates trans interactions between different Greek islands, creating super-enhancer hubs that include neighboring OR clusters ${ }^{38}$. Our pseudotime analysis predicted additionally the co-factor Ssbp2 to play a role in this process. Ssbp2 is in other contexts known to bind to Ldb1 and thereby prevents its degradation by the proteasome $\mathrm{e}^{49,50}$. Thus expression of Ssbp2 in the multigenic phase would amplify the effect of Ldb1. 
All four factors peak during the multigenic phase (Fig. 4A-B, Fig. 5B and SFigure. 9A). This expression is anti-correlated to that of Fos, which supports the competitive interaction hypothesis outlined above (first row of Fig. $5 \mathrm{C}$ ). The high activity of Fos in silent and monogenic phase would counteract complex formation even of the small concentrations of Lhx2 and Ebf1 observed in this stage.

Our results found no OR expression during the Mid.INP stage despite significant expression of Lhx2, Ebf1 and Kdm1a (known activators of OR genes). We note that all components of the polycomb repressive complex 2 (PRC2), Eed, Ezh2 and Suz12, are active in Mid.INP, which could explain the absence of OR gene expression in Mid.INP despite the presence of the activators (second row of Fig. 5C). Moreover, an essential subunit of PRC2, Eed, is significantly reduced during onset of OR expression in Late.INP (Fig. 4 and SFigure 6). This elimination of Eed is sufficient to disassemble the PRC2 complex, which then can no longer maintain the repressive $\mathrm{H} 3 \mathrm{~K} 27 \mathrm{me} 3$ mark $^{52,53}$. Furthermore, we showed a dramatic reduction in expression of Ezh2 and Suz12 subunits of PRC2 along OSN differentiation (second row of Fig. 5C and SFigure 6). We conclude that PRC2 activity may be involved in repression of OR expression in Mid.INP. The disassembly of PRC2 in Late.INP then enables the Greek Island hubs to transiently activate the ciscorresponding OR gene/s, which enables the expression of multiple OR genes in most of Late.INP stage cells at the same time with relatively low levels compared to later stages of OSN differentiation (monogenic phase).

Heterochromatic silencing of ORs throughout OSN differentiation is enforced by the (interchromosomal) convergence of OR loci to OSN-specific, highly compacted nuclear bodies ${ }^{21}$. It has been shown that in the monogenic phase individual active OR genes require de-silencing by lysine demethylase $\mathrm{Kdm} 1 \mathrm{a}^{27}$ and spatial segregation of the single chosen OR allele towards euchromatic nuclear territories ${ }^{21}$. Another study however showed that the deletion of $\mathrm{Kdm} 1$ a leads to persistent multigenic expression, suggesting a silencing role of $\mathrm{Kdm} 1 \mathrm{a}$ rather than activating one $\mathrm{e}^{48}$. Here, pseudotime analysis sheds light on the seemingly contradictory role of $\mathrm{Kdm} 1 \mathrm{a}$ (third row of Fig. $5 \mathrm{C}$ ):

The CBX5 protein is responsible for silencing of OR genes during the silent phase by binding to and thereby protecting the repressive $\mathrm{H} 3 \mathrm{~K} 9 \mathrm{me} 3$ mark in gene bodies ${ }^{21}$. It vanishes upon transition to multigenic phase (SFigure 9B). After H3K9me3 has lost one methyl group (e.g., through the action of $\mathrm{Kdm} 4 \mathrm{a}), \mathrm{Kdm} 1 \mathrm{a}$ can demethylate $\mathrm{H} 3 \mathrm{~K} 9 \mathrm{me} 2 \mathrm{Kdm} 1 \mathrm{a}$ peaks at Mid. INP stage and acts on di-methylated lysines only ${ }^{55}$. Thus the action of Kdm1a on H3K9me3 leads to activation in Mid.INP (Fig. 5C).

In contrast, another methylation site, on H3K4 is a mark of an active promoter/enhancer in the methylated stage (trimethylated for promoter, monomethylated for enhancer). Kdm5a peaks during multigenic phase (Fig. 5C, SFigure 8A) and can demethylate tri- or di-methylated H3K4 to its monomethylated form. Kdm1 a by itself cannot act on H3K4me, but in a complex with Rcor1 and Hdac2 (CoRest complex) it is able to demethylate H3K4me2/1 55,60,61 (Fig. 5C). Rcor1 and Hdac2 have their peak expression during Late.INP stage, i.e. shortly after onset of the Kdm1a peak (Fig. 5B, SFigure 8A). Thus, in Late.INP but not in Mid.INP, Kdm1a can demethylate $\mathrm{H} 3 \mathrm{~K} 4 \mathrm{me} 2 / 1$, resulting in repression. This amounts 
to the multigenic phase (Late.INP) beginning to build up the molecular machinery to downregulate all but one of expressed ORs.

Taken together, the same enzymatic activity of the same factor (demethylation by Kdm1a) results in opposing effects on transcription due to co-factors Rcor1 and Hdac2 modulating substrate specificity of $\mathrm{Kdm} 1 \mathrm{a}$. Moreover our pseudotime analysis supports the hypothesis that OR expression issues a negative feedback signal on $\mathrm{Kdm} 1 \mathrm{a}$ which is mediated by Atf5 and Adcy 3 during transition from multigenic to monogenic phase $25,27,28$ (see SFigure 8B).

During the monogenic phase, a super-enhancer is formed by the trans interaction between multiple Greek islands. Among the OR genes associated with this super-enhancer, merely one OR is expressed at very high levels ${ }^{38}$. During the multigenic phase, $\mathrm{H} 3 \mathrm{~K} 4 \mathrm{me} 3$ marks of Greek islands ${ }^{20}$ are converted to H3K4me, e.g. by Kdm5a (SFigure 5C). The latter histone mark can be recognized by the chromatin reader Zmynd ${ }^{67}$. Zmynd8 has been shown to participate in another, highly specific selection process, namely the expression of $l g h$ genes $^{62}$. There, it recognizes H3K4me and represses super-enhancer activity in B cells. We therefore speculate that it might play a similar role in OR expression.

Expression of Zmynd8 peaks simultaneously with expression of the super-enhancer forming complex Ebf1-Lhx2-Ldb1-Ssbp2 during multigenic phase (Fig. 4C for Zmynd8 and Ssbp2, Fig. 3 for Ebf1 and Lhx2, SFigure 9 for Ldb1). Upon transition to the monogenic phase, Zmynd8 expression ceases while Ebf1 and Sspb2 maintain intermediate expression. The disappearance of the ZMYND8 protein might abolish the suppression of the super-enhancer and could allow very high expression of the selected OR (Fig. 5C bottom row).

So far it is unknown whether the monoallelic expression characteristic for mature OSNs is preceded by a biallelic phase. The dataset evaluated here does not allow us to analyze this question directly, because it does not exhibit enough sequence diversity between alleles to distinguish them. We searched for epigenetic factors known to be involved in allelic selection in other contexts, which show significant and relevant expression changes during OR differentiation. We found two factors, Smchd1 and Cdyl2 (SFigure 6), which were discussed as stabilizing monoallelic expression. They play a role in epigenetic silencing, spermatogenesis, random inactivation of $X$ chromosome, and stabilize monoallelic expression ${ }^{68-70}$. Both factors peak sharply in mid to late INP. Assuming these two factors initiate monoallelic expression, this would place monoallelic selection before or concomitant with the onset of (multigenic) expression in Late.INP, in other words, there might be no biallelic stage at all.

The dissection of the OSN maturation process into different stages allowed us to reveal three phases of OR gene selection. This in turn enabled an in-depth analysis of pseudotime expression profiles ${ }^{71}$, leading to several promising candidates and to testable hypotheses on the mechanisms involved in OR gene selection. It will be interesting to complement the present data with single cell chromatin accessibility data (e.g. scATAC-seq) or single cell chromatin conformation data $(\mathrm{Hi}-\mathrm{C})$ in the context of conditional knockouts of the factors we have identified. Experiments should be carried out in hybrid crosses to 
additionally monitor allelic expression. Our study has narrowed down the cell stages and the time window that need to be analyzed for these purposes, thereby enhancing future research on this topic.

\section{Methods}

Most statistical analysis and visualization were done in RStudio using R version 3.6.3.

\section{a. Data processing and Quality control}

Our analysis of OR expression patterns during OSN differentiation is based on a scRNA-seq dataset generated by (Fletcher et al ${ }^{31}$, GEO: GSE95601). Ngai group investigated the homeostatic differentiation in the postnatal olfactory epithelium. Horizontal basal stem cells (HBC) were released from quiescence by a conditional knockout of the Trp63 transcription factor. Briefly, cells were FACS (fluorescence-activated cell sorting) selected for Sox2-EGFP-pos-itive, ICAM1-negative, SCARB1/F3-negative expression to enrich for the cell population of interest (GBCs, later neuronal intermediates, and microvillous cells over sustentacular cells). Then scRNA-seq was done using the Fluidigm C1 microfluidics cell capture platform followed by Illumina multiplex sequencing. Fletcher et al ${ }^{31}$ used RefSeq transcript annotations to align reads to the GRCm38.3 mouse genome with Tophat2. This resulted in 849 cells with a mean coverage of $1.36 \mathrm{M}$ reads per cell (before read quality control). Contaminations, doublets and other technical artifacts were removed according to the 'oeHBCdiff_filtering.R', protocol (https://github.com/rufletch/p63-HBCdiff).

From here on our data processing differs from Fletcher et al ${ }^{31}$. We included all transcripts (above 40 counts in total) that occured in at least one cell to ensure retrieval even of OR genes with very sporadic expression. Further filtration was done using Seurat (version 3.1.4 $)^{72-74}$, keeping only cells with at least 1250 expressed genes. A total of 687 cells with a mean library size of $460 \mathrm{k}$ unique reads passed all filtration criteria and the median number of genes per cell is 4164 . The library size distribution of these cells is shown in (SFigures 2,3).

Duplicate removal and normalizing the counts was performed by Seurat's SCTransform with default parameters ${ }^{72}$, resulting in a 687 cells times 17228 genes matrix followed by Seurat workflow ${ }^{72}$.

\section{b. Dimension reduction, clustering and cell type assignment}

We followed the Seurat clustering workflow. First, dimension reduction was done using Principal Component Analysis (PCA). The number of principal components kept was set to 15, after assessing the goodness of approximation by JackStraw and ElbowPlot functions. A shared k-nearest neighbor graph was built by the FindNeighbors function. Afterwards, the Lovain algorithm was applied to define 13 distinct clusters from the shared nearest neighbor graph using the FindClusters function and the Jaccard index as a similarity measure. This number matches the number of clusters identified in (Fletcher et $\mathrm{al}^{31}$ ) 
for this data. The expression of cell type marker genes that were collected from the literature (STable 1) served to assign cell clusters manually to known cell types according to the Seurat guidelines ${ }^{72}$. At least two expressed markers were required to confidently annotate a specific cell type. Visualization of the data was performed by PCA, UMAP ${ }^{75}$ and $\mathrm{tSNE}^{76}$.

\section{c. Trajectory inference and pseudotime assignment}

Slingshot ${ }^{36}$ was used to construct a minimum spanning tree (MST) based on the 15-dimensional representation of the cells obtained above. The topology of the MST is independent of the root choice. For biological reasons, we selected qGBC as the root for the assignment of pseudo time, where it can differentiate to any cell type of $\mathrm{MOE}^{35}$. For each cell, a pseudotime between 0 (cells at the root node) and 1 (leaf node cells) was assigned by the slingPseudotime function.

\section{d. Differential expression analysis}

For each cell stage, we identified marker genes showing differential expression compared to all other cell stages using FindAllMarkers in Seurat, using the Wilcoxon rank sum test. Supplemental Fig. 3 shows a heatmap of the top 10 differentially expressed genes (i.e., putative markers) for each cell stage of MOE.

Among 2712 (co-)TFs (include chromatin remodelers) obtained from the GO.db package and AnimalTFDB3.0 (http://bioinfo.life.hust.edu.cn/AnimalTFDB/\#!/), we found 2004 (co-)TFs expressed (at least one count in one cell) in the neuronal lineage. In later differential expression analysis we compared the expression profiles of cell stages that were placed consecutively along the neuronal trajectory (i.e., the maturation of OSN) to identify genes that change their expression upon transition between cell stages (SFigure 4B). Volcano plots for each cell type transition were generated by a slightly modified EnhancedVolcano function (https://github.com/kevinblighe/EnhancedVolcano).

Differential expression analysis for silent to multigenic and multigenic to monogenic phase transitions were performed by comparison of pre-Late.INP cells vs. Late.INP cells and Late.INP vs. post-Late.INP cells, respectively. Genes with a Bonferroni adjusted p-value $<0.05$ (Wilcoxon rank sum test, FindMarkers function in Seurat) and an average absolute $F C>=2$ were considered differentially expressed. This yielded 83 respectively 39 differentially expressed (co-)TFs for the two transitions.

\section{e. Motif Analysis}

The genomic ranges of 68 OR clusters and 63 Greek islands were compiled from Monahan et al ${ }^{11}$, which allows the matching of OR clusters and Greek islands. UCSC genome browser tools were used to find all genes inside OR clusters. We performed a motif search on the 63 Greek island sequences (Excel file 7) and the approximate promoter regions (500bp upstream the transcription starting sites) of all OR genes 
were obtained by using the "ucsc-twobittofa" bioconda package and the "biomart" R package respectively. The MEME suite web server for motif search and analysis ${ }^{40,41}$ was used to predict the transcription factors (TF) that bind to Greek islands. We applied MEME using default values for all parameters to find the novel, ungapped motifs inside Greek islands with the following command: meme greek_islands.fa dna -oc. -nostatus -time 14400 -mod zoops -nmotifs 3 -minw 6 -maxw 50 -objfun classic -revcomp markov_order 0

Then we performed motif comparison between each motif found in the above-mentioned analysis against a database of known TFs motifs (JASPAR2018_CORE_non-redundant and uniprobe_mouse databases) using Tomtom too ${ }^{42}$. The Pearson correlation coefficient was used to measure the similarity between columns of position weight matrices (PWMs) and we restricted the results by setting q-value $<=$ 0.1 (rather than 0.5 by default) as a threshold (10\% FDR) using the following command: tomtom -no-ssc oc. -verbosity 1 -min-overlap 5 -mi 1 -dist pearson -thresh 0.1 -time 300 query_motifs $\mathrm{db} /$ MOUSE/uniprobe_mouse.meme db/JASPAR/JASPAR2018_CORE_non-redundant.meme

We also investigated the enrichment motifs in 63 Greek islands sequences using AME tool ${ }^{46}$ by using an average odds score method and fisher's exact test as a motif enrichment test through the following command: ame --verbose 1 -oc. -scoring avg -method fisher -hit-lo-fraction 0.25 -evalue-report-threshold 1.0 --control -shuffle- -kmer 2 greek_islands.fa db/MOUSE/uniprobe_mouse.meme

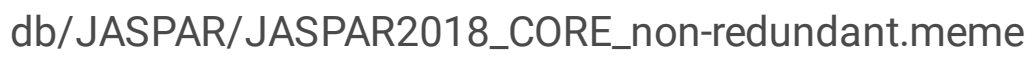

Finally, a strict motif search in Greek islands for selected TFs was done by "ucsc-findmotif" bioconda package, allowing for 3 mismatches.

\section{f. Visualization of time series}

Grouped time series ${ }^{77}$ was used to visualize pseudotime series of individual genes and to calculate and visualize aggregated groups of genes, e.g. all OR genes. Since the original expression count matrix is sparse (75.45\% zero count entries), we first applied ALRA ${ }^{78}$, which has specifically been designed for the imputation of missing values in scRNA-Seq data (SFigure 3). The imputed expression matrix retrieved $\sim 2403$ missing values, reducing the fraction of zero count entries to $61.50 \%$. The median number of expressed genes per cell was 6715 (see SFigure 2,3). Note that the imputed expression matrix was used only for visualization, for all analysis steps we used normalized counts without data imputation.

\section{Declarations}

\section{Author contribution statement}

A.T. and S.I.K. conceived and designed the analysis; M.H. collected the data, conducted the analysis and prepared the figures. All authors wrote, read and approved the manuscript. 


\section{Data availability statement}

The single cell data has been generated by ${ }^{31}$ and is available at GEO under the accession number GSE95601[ https://www.ncbi.nlm.nih.gov/geo/query/acc.cgi?acc=GSE95601 ]. The processed data and all additional data used in our analysis are available as Supplemental Materials.

\section{Additional information}

The authors declare no competing interests.

\section{References}

1. Korsching, S. I. Olfaction. in The Physiology of Fishes 256, Chapter 14 (CRC Press, 2020).

2. Niimura, Y. Olfactory receptor multigene family in vertebrates: from the viewpoint of evolutionary genomics. Curr. Genomics 13, 103-114 (2012).

3. Buck, L. \& Axel, R. A novel multigene family may encode odorant receptors: a molecular basis for odor recognition. Cell 65, 175-187 (1991).

4. Chess, A., Simon, I., Cedar, H. \& Axel, R. Allelic inactivation regulates olfactory receptor gene expression. Cell 78, 823-834 (1994).

5. Mombaerts, P. et al. Visualizing an Olfactory Sensory Map. Cell 87, 675-686 (1996).

6. Ressler, K. J., Sullivan, S. L. \& Buck, L. B. Information coding in the olfactory system: Evidence for a stereotyped and highly organized epitope map in the olfactory bulb. Cell 79, 1245-1255 (1994).

7. Buck, L. B. Information Coding in the Vertebrate Olfactory System. Annu. Rev. Neurosci. 19, 517-544 (1996).

8. Feinstein, P. \& Mombaerts, P. A Contextual Model for Axonal Sorting into Glomeruli in the Mouse Olfactory System. Cell 117, 817-831 (2004).

9. Serizawa, S., Miyamichi, K. \& Sakano, H. One neuron-one receptor rule in the mouse olfactory system. Trends Genet. 20, 648-653 (2004).

10. Mombaerts, P. Odorant receptor gene choice in olfactory sensory neurons: the one receptor-one neuron hypothesis revisited. Curr. Opin. Neurobiol. 14, 31-36 (2004).

11. Monahan, K. et al. Cooperative interactions enable singular olfactory receptor expression in mouse olfactory neurons. eLife 6, e28620 (2017).

12. Zhang, X. et al. High-throughput microarray detection of olfactory receptor gene expression in the mouse. Proc. Natl. Acad. Sci. 101, 14168-14173 (2004).

13. Miyamichi, K., Serizawa, S., Kimura, H. M. \& Sakano, H. Continuous and Overlapping Expression Domains of Odorant Receptor Genes in the Olfactory Epithelium Determine the Dorsal/Ventral Positioning of Glomeruli in the Olfactory Bulb. J. Neurosci. 25, 3586-3592 (2005). 
14. Tsuboi, A., Miyazaki, T., Imai, T. \& Sakano, H. Olfactory sensory neurons expressing class I odorant receptors converge their axons on an antero-dorsal domain of the olfactory bulb in the mouse. Eur. J. Neurosci. 23, 1436-1444 (2006).

15. Markenscoff-Papadimitriou, E. et al. Enhancer Interaction Networks as a Means for Singular Olfactory Receptor Expression. Cel/ 159, 543-557 (2014).

16. Michaloski, J. S., Galante, P. A. F. \& Malnic, B. Identification of potential regulatory motifs in odorant receptor genes by analysis of promoter sequences. Genome Res. 16, 1091-1098 (2006).

17. Hirota, J. \& Mombaerts, P. The LIM-homeodomain protein Lhx2 is required for complete development of mouse olfactory sensory neurons. Proc. Natl. Acad. Sci. 101, 8751-8755 (2004).

18. Clowney, E. J. et al. High-throughput mapping of the promoters of the mouse olfactory receptor genes reveals a new type of mammalian promoter and provides insight into olfactory receptor gene regulation. Genome Res. 21, 1249-1259 (2011).

19. Plessy, C. et al. Promoter architecture of mouse olfactory receptor genes. Genome Res. 22, 486-497 (2012).

20. Magklara, A. et al. An Epigenetic Signature for Monoallelic Olfactory Receptor Expression. Cell 145, 555-570 (2011).

21. Clowney, E. J. et al. Nuclear Aggregation of Olfactory Receptor Genes Governs Their Monogenic Expression. Cell 151, 724-737 (2012).

22. Lyons, D. B. et al. Heterochromatin-Mediated Gene Silencing Facilitates the Diversification of Olfactory Neurons. Cell Rep. 9, 884-892 (2014).

23. Armelin-Correa, L. M., Gutiyama, L. M., Brandt, D. Y. C. \& Malnic, B. Nuclear compartmentalization of odorant receptor genes. Proc. Natl. Acad. Sci. 111, 2782-2787 (2014).

24. Armelin-Correa, L. M., Nagai, M. H., Silva, A. G. L. \& Malnic, B. Nuclear architecture and gene silencing in olfactory sensory neurons. BioArchitecture 4, 160-163 (2014).

25. Serizawa, S. et al. Negative feedback regulation ensures the one receptor-one olfactory neuron rule in mouse. Science 302, 2088-2094 (2003).

26. Lewcock, J. W. \& Reed, R. R. A feedback mechanism regulates monoallelic odorant receptor expression. Proc. Natl. Acad. Sci. 101, 1069-1074 (2004).

27. Lyons, D. B. et al. An Epigenetic Trap Stabilizes Singular Olfactory Receptor Expression. Cell 154, 325-336 (2013).

28. Dalton, R. P., Lyons, D. B. \& Lomvardas, S. Co-Opting the Unfolded Protein Response to Elicit Olfactory Receptor Feedback. Cell 155, 321-332 (2013).

29. Hanchate, N. K. et al. Single-cell transcriptomics reveals receptor transformations during olfactory neurogenesis. Science (2015) doi:10.1126/science.aad2456.

30. Tan, L., Li, Q. \& Xie, X. S. Olfactory sensory neurons transiently express multiple olfactory receptors during development. Mol. Syst. Biol. 11, 844 (2015). 
31. Fletcher, R. B. et al. Deconstructing Olfactory Stem Cell Trajectories at Single-Cell Resolution. Cell Stem Cel/ 20, 817-830.e8 (2017).

32. Caggiano, M., Kauer, J. S. \& Hunter, D. D. Globose basal cells are neuronal progenitors in the olfactory epithelium: A lineage analysis using a replication-incompetent retrovirus. Neuron 13, 339-352 (1994).

33. Chen, X., Fang, H. \& Schwob, J. E. Multipotency of purified, transplanted globose basal cells in olfactory epithelium. J. Comp. Neurol. 469, 457-474 (2004).

34. Chen, M. et al. Wnt-Responsive Lgr5+ Globose Basal Cells Function as Multipotent Olfactory Epithelium Progenitor Cells. J. Neurosci. 34, 8268-8276 (2014).

35. Jang, W., Chen, X., Flis, D., Harris, M. \& Schwob, J. E. Label-retaining, quiescent globose basal cells are found in the olfactory epithelium. J. Comp. Neurol. 522, 731-749 (2014).

36. Street, K. et al. Slingshot: cell lineage and pseudotime inference for single-cell transcriptomics. BMC Genomics 19, 477 (2018).

37. Monahan, K. \& Lomvardas, S. Monoallelic Expression of Olfactory Receptors. Annu. Rev. Cell Dev. Biol. 31, 721-740 (2015).

38. Monahan, K., Horta, A. \& Lomvardas, S. LHX2- and LDB1-mediated trans interactions regulate olfactory receptor choice. Nature 565, 448-453 (2019).

39. Lomvardas, S. et al. Interchromosomal Interactions and Olfactory Receptor Choice. Cell 126, 403413 (2006).

40. Bailey, T. L. et al. MEME Suite: tools for motif discovery and searching. Nucleic Acids Res. 37, W202W208 (2009).

41. Bailey, T. L., Johnson, J., Grant, C. E. \& Noble, W. S. The MEME Suite. Nucleic Acids Res. 43, W39W49 (2015).

42. Gupta, S., Stamatoyannopoulos, J. A., Bailey, T. L. \& Noble, W. S. Quantifying similarity between motifs. Genome Biol. 8, R24 (2007).

43. Wang, H.-N. et al. Inhibition of c-Fos expression attenuates IgE-mediated mast cell activation and allergic inflammation by counteracting an inhibitory AP1/Egr1/IL-4 axis. J. Transl. Med. 19, 261 (2021).

44. Ray, N. et al. c-Fos suppresses systemic inflammatory response to endotoxin. Int. Immunol. 18, 671677 (2006).

45. Bahrami, S. \& Drabløs, F. Gene regulation in the immediate-early response process. Adv. Biol. Regul. 62, 37-49 (2016).

46. McLeay, R. C. \& Bailey, T. L. Motif Enrichment Analysis: a unified framework and an evaluation on ChIP data. BMC Bioinformatics 11, 165 (2010).

47. Zhang, G., Titlow, W. B., Biecker, S. M., Stromberg, A. J. \& McClintock, T. S. Lhx2 Determines Odorant Receptor Expression Frequency in Mature Olfactory Sensory Neurons. eNeuro 3, (2016). 
48. Vyas, R. N., Meredith, D. \& Lane, R. P. Lysine-specific demethylase-1 (LSD1) depletion disrupts monogenic and monoallelic odorant receptor (OR) expression in an olfactory neuronal cell line. Mol. Cell. Neurosci. 82, 1-11 (2017).

49. Wang, Y. et al. SSBP2 is an in vivo tumor suppressor and regulator of LDB1 stability. Oncogene 29, 3044-3053 (2010).

50. Wang, H. et al. Crystal structure of human LDB1 in complex with SSBP2. Proc. Natl. Acad. Sci. 117, 1042-1048 (2020).

51. Wu, Y. et al. Structure of the MADS-box/MEF2 Domain of MEF2A Bound to DNA and Its Implication for Myocardin Recruitment. J. Mol. Biol. 397, 520-533 (2010).

52. Cao, Q. et al. The central role of EED in the orchestration of polycomb group complexes. Nat. Commun. 5, 3127 (2014).

53. Potjewyd, F. et al. Degradation of Polycomb Repressive Complex 2 with an EED-Targeted Bivalent Chemical Degrader. Cell Chem. Biol. 27, 47-56.e15 (2020).

54. Qiao, Q. et al. The Structure of NSD1 Reveals an Autoregulatory Mechanism Underlying Histone H3K36 Methylation. J. Biol. Chem. 286, 8361-8368 (2011).

55. Bannister, A. J. \& Kouzarides, T. Regulation of chromatin by histone modifications. Cell Res. 21, 381395 (2011).

56. Wang, J. et al. Opposing LSD1 complexes function in developmental gene activation and repression programmes. Nature 446, 882-887 (2007).

57. Kilinc, S., Savarino, A., Coleman, J. H., Schwob, J. E. \& Lane, R. P. Lysine-specific demethylase-1 (LSD1) is compartmentalized at nuclear chromocenters in early post-mitotic cells of the olfactory sensory neuronal lineage. Mol. Cell. Neurosci. 74, 58-70 (2016).

58. Rusconi, F., Grillo, B., Toffolo, E., Mattevi, A. \& Battaglioli, E. NeuroLSD1: Splicing-Generated Epigenetic Enhancer of Neuroplasticity. Trends Neurosci. 40, 28-38 (2017).

59. Coleman, J. H., Lin, B. \& Schwob, J. E. Dissecting LSD1-Dependent Neuronal Maturation in the Olfactory Epithelium. J. Comp. Neurol. 525, 3391-3413 (2017).

60. Song, Y. et al. Mechanism of Crosstalk between the LSD1 Demethylase and HDAC1 Deacetylase in the CoREST Complex. Cell Rep. 30, 2699-2711.e8 (2020).

61. Shi, Y.-J. et al. Regulation of LSD1 Histone Demethylase Activity by Its Associated Factors. Mol. Cell 19, 857-864 (2005).

62. Delgado-Benito, V. et al. The Chromatin Reader ZMYND8 Regulates Igh Enhancers to Promote Immunoglobulin Class Switch Recombination. Mol. Cell 72, 636-649.e8 (2018).

63. Lin, C., Garruss, A. S., Luo, Z., Guo, F. \& Shilatifard, A. The RNA Pol II Elongation Factor Ell3 Marks Enhancers in ES Cells and Primes Future Gene Activation. Cell 152, 144-156 (2013).

64. Levine, S. S. et al. The Core of the Polycomb Repressive Complex Is Compositionally and Functionally Conserved in Flies and Humans. Mol. Cell. Biol. (2002) doi:10.1128/MCB.22.17.60706078.2002 . 
65. Vandamme, J., Völkel, P., Rosnoblet, C., Faou, P. L. \& Angrand, P.-O. Interaction Proteomics Analysis of Polycomb Proteins Defines Distinct PRC1 Complexes in Mammalian Cells *. Mol. Cell. Proteomics 10, (2011).

66. Shirato, H. et al. A Jumonji (Jarid2) Protein Complex Represses cyclin D1 Expression by Methylation of Histone H3-K9 *. J. Biol. Chem. 284, 733-739 (2009).

67. Li, N. et al. ZMYND8 reads the dual histone mark H3K4me1-H3K14ac to antagonize the expression of metastasis-linked genes. Mol. Cell 63, 470-484 (2016).

68. Mould, A. W. et al. Smchd1 regulates a subset of autosomal genes subject to monoallelic expression in addition to being critical for X inactivation. Epigenetics Chromatin 6, 19 (2013).

69. Qin, R. et al. CDYL Deficiency Disrupts Neuronal Migration and Increases Susceptibility to Epilepsy. Cell Rep. 18, 380-390 (2017).

70. Liu, S. et al. Chromodomain Protein CDYL Acts as a Crotonyl-CoA Hydratase to Regulate Histone Crotonylation and Spermatogenesis. Mol. Cell 67, 853-866.e5 (2017).

71. Tanay, A. \& Regev, A. Scaling single-cell genomics from phenomenology to mechanism. Nature 541, $331-338$ (2017).

72. Satija, R., Farrell, J. A., Gennert, D., Schier, A. F. \& Regev, A. Spatial reconstruction of single-cell gene expression data. Nat. Biotechnol. 33, 495-502 (2015).

73. Hafemeister, C. \& Satija, R. Normalization and variance stabilization of single-cell RNA-seq data using regularized negative binomial regression. Genome Biol. 20, 296 (2019).

74. Stuart, T. \& Satija, R. Integrative single-cell analysis. Nat. Rev. Genet. 20, 257-272 (2019).

75. Mclnnes, L., Healy, J., Saul, N. \& Großberger, L. UMAP: Uniform Manifold Approximation and Projection. J. Open Source Softw. 3, 861 (2018).

76. Maaten, L. van der \& Hinton, G. Visualizing Data using t-SNE. J. Mach. Learn. Res. 9, 2579-2605 (2008).

77. Hyndman, R. \& Athanasopoulos, G. Forecasting: Principles and Practice. (2021).

78. Linderman, G. C., Zhao, J. \& Kluger, Y. Zero-preserving imputation of scRNA-seq data using low-rank approximation. bioRxiv 397588 (2018) doi:10.1101/397588.

\section{Figures}

\section{Figure 1}

Cell type identification, trajectory inference and pseudotime assignment. A) 2D PCA projection of MOE cells shows the four predicted lineages starting from qGBC (center, pink, the starting point of the four trajectories is marked in green). The differentiation trajectory end points (red dots at the end of paths) are HBCO (top left, pale blue), mSus (center left, green), MV (bottom, brown) and mOSN (top right, purple). 
The transition stages in the four lineages (black dots) are HBC1 (light blue), HBC2 (blue), iSus (light green), GBC (red), Early.INP (pale orange), Mid.INP (light orange), Late.INP (dark orange) and iOSN (light purple) from the left to the right. B) DotPlot representation of the average expression (red for high expression and pale blue for lower one) of known marker genes (x.axis) corresponding to cell types of MOE (y.axis), and the size of each dot represents the percentage of cells that expresses a corresponding marker gene in a given cell type.

\section{Figure 2}

OR expression dynamics along neuronal lineage. A) Aggregate expression (normalized pseudocounts) of all OR genes for each cell. Cells are sorted according to pseudotime and colored according to cell type. B) Number of active OR clusters per cell, sorted by pseudotime. C) Expression of the OR gene with the highest expression level in each cell ("winner"). For each stage of the neuronal lineage, we show the distribution of the corresponding expression values (pseudocounts) as a violin plot. D) Using the same representation as in $\mathrm{C}$, the expression of the OR gene with second, third and fourth highest expression ("runners up" 1-3) in each cell is shown in the top, middle, and bottom row, respectively. Note that the yaxis is in log scale, and that the scale of the winner expression is on average one to two orders of magnitude higher than that of the runners up. The dotted horizontal line in $\mathbf{C}$ ) and $\mathbf{D}$ ) marks an expression level of 50 counts.

\section{Figure 3}

Greek islands binding motifs and representative pseudotime courses of respective TFs. A) Binding motifs found in Greek islands. The top row shows the motif found de novo. In the rows below, known binding sites of transcription factors that partly align with the de novo motif in different sites (TOMTOM) and/or are enriched in Greek islands (AME). The transcription factors given in square brackets refer to the TFs found only by Tomtom alignment whereas the round brackets refer to TFs found only by forward motif search (see SFigure 5). B) Single cell pseudotime courses of the TFs in bold print show characteristic trends along OSN differentiation. The grouping of the TFs in gray follows their pseudotime profile. It agrees with their grouping according to binding sites, except for Pax6 and Pou2f1 that have a homeobox domain like Lhx2.

\section{Figure 4}

Differential expression analysis and pseudotime expression profiles of candidate factors. A) Volcano plots revealing the up- (peach) and downregulated (magenta) factors in the two transitions from silent to 
multigenic (left) and from multigenic to monogenic phase (right). TFs are shown as filled circles, cofactors are triangles. We selected (co-)TFs* with an adjusted p-value less than 0.05 (y-axis, log10 Bonferroni adjusted $p$-value) and an average expression change of at least 2 fold (x.axis, log2 fold). (Co-)TFs with only significant changes are shown in brown, those with only relevant expression fold $>2$ are colored in grey, all others are in black (not significant). B) Single cell pseudotime courses of selected transcription factors and cofactors that show characteristic trends along OSN differentiation (cell stages are indicated by colors). Left to right: each group of 2 columns shows examples of transcription (co-)factors in group 1, 2, and 3 as defined in the text. C) Same as B) for chromatin remodelers. *Note that co-TFs in A) include chromatin remodelers.

\section{Figure 5}

Graphical summary of OR gene expression and hypothetical selection mechanisms. A) Frequency distribution of active OR clusters and chromosomes per cell in the three phases of OR expression. Two exceptional cases of cells in multigenic phase with 6 respectively 7 active OR clusters are omitted from the plot. B) Pseudotime ordering of (co-)TFs and remodelers according to peak of expression in one of the three phases. Factors that so far have not been reported are highlighted in red. C) Four mechanisms potentially contributing to OR selection. Factors are colored according to their phase of peak expression. The placement of the nucleosomes is according to the pseudotime at which the respective processes take place. Green arrows indicate an activating effect on target genes, black blunt end arrows indicate an inhibitory effect. Top row: Competitive binding of Fos vs. Ebf1 and Lhx2 to different parts of the Greek island consensus motif, resulting in different enhancer activities during the three OR selection phases. Second row: Downregulation of the Eed subunit dissolves the PRC2 complex at the beginning of the multigenic phase and thus enables access of demethylases to H3K27me3. Third row: Methylated H3K9 is recognized, bound and protected by $\mathrm{Cbx} 5$ during the silent phase. At the end of this phase $\mathrm{Cb} \times 5$ is downregulated and $\mathrm{Kdm} 1 \mathrm{a}$ is upregulated. It participates in the process of $\mathrm{H} 3 \mathrm{~K} 9$ demethylation by removing the H3K9me1/2 mark. Later, after H3K4me3 has been demethylated to H3K4me2, presumably by $\mathrm{Kdm} 5 \mathrm{a}$ during the multigenic phase, $\mathrm{Kdm} 1 \mathrm{a}$ has a second role as part of the CoRest complex and demethylates H3K4me2. Bottom row: After demethylation of H3K4me3/2 by Kdm5a at Greek island enhancers, the chromatin reader Zmynd8, which peaks during the multigenic phase, can recognize H3K4me and repress enhancer activity. Activity is restored upon downregulation of Zmynd8 in monogenic phase.

\section{Supplementary Files}

This is a list of supplementary files associated with this preprint. Click to download.

- SupplementaryFiles.zip 